\title{
A Simulation Tool for Hurricane Evacuation Planning
}

\author{
Daniel J. Fonseca, ${ }^{1}$ Gary P. Moynihan, ${ }^{2}$ Jordan Johnston, ${ }^{3}$ and Jordan Jennings ${ }^{3}$ \\ ${ }^{1}$ Department of Mechanical Engineering, The University of Alabama, Tuscaloosa, AL 35487-0276, USA \\ ${ }^{2}$ Department of Civil, Construction and Environmental Engineering, The University of Alabama, Tuscaloosa, AL 35487, USA \\ ${ }^{3}$ Department of Industrial Engineering, The University of Alabama, Tuscaloosa, AL 35487, USA
}

Correspondence should be addressed to Daniel J. Fonseca, dfonseca@eng.ua.edu

Received 17 February 2009; Revised 20 August 2009; Accepted 15 December 2009

Recommended by Evtim Peytchev

\begin{abstract}
Atlantic hurricanes and severe tropical storms are a serious threat for the communities in the Gulf of Mexico region. Such storms are violent and destructive. In response to these dangers, coastal evacuation may be ordered. This paper describes the development of a simulation model to analyze the movement of vehicles through I-65, a major US Interstate highway that runs north off the coastal City of Mobile, Alabama, towards the State of Tennessee, during a massive evacuation originated by a disastrous event such a hurricane. The constructed simulation platform consists of a primary and two secondary models. The primary model is based on the entry of vehicles from the 20 on-ramps to I-65. The two secondary models assist the primary model with related traffic events such as car breakdowns and accidents, traffic control measures, interarrival signaling, and unforeseen emergency incidents, among others. Statistical testing was performed on the data generated by the simulation model to indentify variation in relevant traffic variables affecting the timely flow of vehicles travelling north. The performed statistical analysis focused on the closing of alternative on-ramps throughout the Interstate.
\end{abstract}

Copyright ( 92009 Daniel J. Fonseca et al. This is an open access article distributed under the Creative Commons Attribution License, which permits unrestricted use, distribution, and reproduction in any medium, provided the original work is properly cited.

\section{Introduction}

The Gulf Coast of Alabama is periodically subjected to severe tropical storms and hurricanes. As an increasing number of Alabama's population lives in the eight counties closest to the Gulf of Mexico, a considerable amount of the State's population is becoming more vulnerable to this type of extreme weather events [1].

The normal response to these occurrences is to evacuate inland from the coast. Normal traffic flows turn into congestion, frustration, and gridlock. This reduces the number of vehicles that can leave the Gulf area if an evacuation order is given. The potential risk for loss of life increases if the hurricane strikes stalled traffic, as people's efforts to evacuate might place them at greater risk than they would have faced if they had stayed out.

In response to Hurricane Floyd (September 1999), extensive traffic delays occurred along inland evacuation routes throughout the state of Florida. Subsequently, FEMA conducted regional meetings to identify approaches for better traffic planning, management, and coordination. A variety of simulation tools were applied to project traffic flows during extreme events requiring evacuation. For example, Pal et al. [2] applied the Oak Ridge Evacuation Modeling System (OREMS) to evaluate traffic conditions resulting from evacuation from Baldwin and Mobile counties in Alabama.

Such hurricane evacuation planning led to the State of Alabama's initial plan to "reverse-lane" I-65 to expedite such evacuations from the Gulf Coast. "Reverse-laning" is the process of reversing one direction of traffic on specific routes to facilitate overall evacuation flow. This procedure is generally applicable to Interstates, referred to as "denied access routes", since traffic control can be applied to interchanges and terminal areas. In this case, I-65 would be reverse-laned such that all traffic would flow north, from south of Alabama Route 225, near Mobile, to Exit 167/168, just south of the greater Montgomery metropolitan area [3]. This concept was further refined after Hurricane Katrina, to provide emergency vehicles with an alternate route south via U.S. 31. 
The I-65 evacuation route has been subject to considerable analysis (e.g., [2, 4]). Yet these analyses have focused only on capacity and congestion issues relating to I-65, itself. This route is part of a larger network of state and county roads flowing north from the Gulf Coast and forming an interdependent system of traffic flow which thus affects the efficiency of the reverse-laned interstate. It has been suggested that selective control of specific on-ramps may improve the effectiveness of the overall evacuation routes. For example, prioritization could be based on level of danger (giving people living closest to the coast priority access to I-65, with other communities directed to other state roads). These planning alternatives could be evaluated through a series of automated sensitivity analyses via discrete simulation.

\section{Road Evacuation Dynamics}

Massive terrestrial evacuation can be seen as a network optimization problem aiming at selecting optimal routes from a set of candidate roads within an existing evacuation network. This selection involves determining where the potential evacuation routes' origin and destination points are located, their maximum traffic volumes, and the type of evacuation schemes resulting in a maximum vehicle exit rate with minimal travel times [5-8].

A commonly used objective function in the road network evacuation problem is the minimization of the total cost originated from the massive traveling on any link. An alternative criterion is the establishment of an objective function which maximizes the ease of vehicle evacuation while minimizing the risk of not evacuating individuals under eminent danger fast enough. This relates to the travel time in a highly congested, constrained network. Leblanc [5] developed a model for analyzing road transportation systems through a time-constrained network model where the time along any road for each user is defined via the average travel time function. In a congested network, the travel time function depends upon the travel demand that tends to be a nonlinear function of the total flow along the road. The sum of all users' travel times along the different links is known as the total travel time function.

A relevant issue while managing a disaster evacuation operation is the pattern of flow in the roadways, that is, equilibrium or nonequilibrium flow. The equilibrium network is satisfied when the distribution of flow in the network is obedient to the Wardrop's principles [9-11]. Such principles imply that the total flow of evacuating vehicles eventually reaches an equilibrium state in which every car has the same travel time. In contrast, a flow pattern in a nonequilibrium flow model cannot satisfy flow conversation constraints since each vehicle uses a distinct route depending upon the overall evacuation strategy devised [12].

In addition, the network evacuation problem can be analyzed as discrete or continuous network repetitions. Discrete network analysis emphasizes on searching evacuation scenarios in terms of capacity enhancements [12]. The objective of the analysis is to choose those roads to be included in the evacuation network, accounting for the effects that such a decision may have on the volume of traffic leaving the area under distress. On the other hand, continuous network modeling focuses on maximizing the capacity expansion of existing, predetermined evacuation networks.

However, during a hurricane evacuation, where the massive exodus of vehicles takes place within a relatively short span of time, the inherent traffic network moves from overcongestion to oversaturation. Under oversaturation conditions, traffic flow optimization is not feasible due to the overwhelming network inflow rate as compared to the exiting rate; thus, the utilization of computer-based simulation is a more appropriate means of modeling the complexity of the flow pattern.

The evacuation network involved is this study corresponds to a discrete network presenting a flow pattern in equilibrium. The network consists of a major US Interstate highway with a set of 20 available on-ramp exits. The closing or opening of these ramps to traffic bound north represents the main decision variable of the analysis.

\section{Approach}

Review of the literature has noted considerable work on the development of decision rules and computer-based support systems to aid in decision-making [13]. A variety of mathematical models have been developed which focus on evacuation route planning. Network representations of evacuation problems are extensions of the classical operations research assignment problem. For these problems, the basic form of the network is that of the more general minimal cost transshipment (or flow) network. In the network, the arcs represent the flow of people, the source nodes represent initial source inventories (points of entrance into the evacuation network), and the sink nodes represent the final inventories (in this case, destinations). Optimization models (e.g., linear programming, goal programming, or dynamic programming) are another category of mathematical models. The model is formulated to either maximize or minimize the objective function (depending upon the purpose of the model) within the context of available resources and constraints [14].

Monte Carlo simulation via discrete simulation was considered for projecting the uncertainties in traffic flow during the study. Braess's paradox mathematical model was originally developed regarding the congestion of signals in transmission networks $[15,16]$. The cited paradox was discovered in 1968 by Dietrich Braess, who found out that increased capacity in congested electronic networks slows down communication. During the past decades, several authors have focused their efforts in understanding the implications of Braess's paradox. They have developed heuristics and mathematical models to predict and explain why this counterintuitive situation occurs. However, this knowledge has not been used by highway officials. There is evidence of Braess's paradox in newly constructed roads around the world, as in the case cited in the literature of a road built in Stuttgart, Germany, which deteriorated traffic conditions in Schlossplatz to a point where it had to be closed 
TABle 1: Counties included in the study.

\begin{tabular}{lcc}
\hline County & Pouulatlon & Persons/vehicle \\
\hline Mobile & 400526 & 0.96 \\
Washinqton & 17906 & 0.73 \\
Baldwin & 156701 & 0.73 \\
Escambia & 38336 & 0.96 \\
Conecuh & 13453 & 0.855 \\
Monroe & 23725 & 0.96 \\
Butler & 20764 & 1.205 \\
Lowndes & 13210 & 1.205 \\
\hline
\end{tabular}

down [17]. The major obstacle for the application of the mentioned heuristics and mathematical models to improve traffic conditions in congested highways, as it is the case during a massive evacuation event, is the lack of knowledge on the premises of Braess's paradox. Subsequent work by Fonseca et al. [18] demonstrated that this could be extended to traffic analysis in a small city. Investigation was conducted regarding the further application of this approach to better project traffic congestion due to hurricane evacuation from the Gulf coast.

This led to an overall proof of the selected simulation concept, initially focusing on management of entry ramps, applied to selected areas of the I-65 extended network. The study involved eight counties and twenty entry ramps along I-65. Table 1 depicts information on the counties' populations and their corresponding persons-vehicle ratios. Table 2 shows the estimates of the traffic volumes emerging from each on the 20 selected ramps during an eventual hurricane evacuation. These data were obtained with the help of the Alabama Department of Transportation and the Alabama Demographics Center. Figure 1 illustrates the geographic location of the selected counties along the Interstate 65.

As previously mentioned, a discrete simulation model was developed to assess the effect of closing selective ramps on the overall traffic evacuation rate, that is, the number of vehicles evacuated from the area in question per hour. The idea behind the analysis is that having all ramps open to vehicles exiting the region might actual be detrimental for the overall evacuation effort since in-flow congestion may be generated by entry ramps located within a few miles from each other and due to the difficulty encountered by emergency and law-enforcement vehicles bound South when all ramps are exclusively for north-bound traffic.

Accepted development methodology identifies five primary phases: (1) data acquisition, (2) system design, (3) system construction, (4) verification and validation, and (5) experimentation and analysis [19]. During the data acquisition phase, the key concepts, relationships, and algorithms were identified. For example, the ALDOT traffic database [20] was investigated to obtain traffic data on interstate and state highways. The evacuation model was constructed via a discrete simulation software, Arena, which was interfaced with Excel macros for data input processing. Arena, a product of Rockwell Automation, is a software tool that
TABLE 2: Exits locations and traffic values.

\begin{tabular}{lcc}
\hline Exit numher & Exit location & Total vehicles \\
\hline 22 & Washinqton Mobile & 441743 \\
31 & Baldwin & 53664 \\
34 & Baldwin & 53664 \\
37 & Baldwin & 53664 \\
45 & Baldwin & 53664 \\
54 & Escambia & 9983 \\
57 & Escambia & 9983 \\
69 & Escambia & 9983 \\
77 & Escambia & 9983 \\
83 & Conecuh Monroe & 10112 \\
93 & Conecuh Monroe & 10112 \\
96 & Conecuh Monroe & 10112 \\
101 & Conecuh Monroe & 10112 \\
107 & Butler & 4307 \\
114 & Butler & 4307 \\
128 & Butler & 4307 \\
130 & Butler & 4307 \\
142 & Lowndes & 3654 \\
151 & Lowndes & 3654 \\
158 & Lowndes & 3654 \\
\hline
\end{tabular}

combines both high-level modeling with general-purpose procedural programming. The software is provided with interchangeable templates of graphical simulation objects as well as statistical data analysis modules that offer a mixture of model building and statistical assessment devices ([21], 2007).

The models of each devised scenario were run at least 30 times to ensure the soundness of the statistical analyses performed. The general simulation framework is discussed in Section 4.

\section{The Simulation Model}

The constructed hurricane evacuation simulation for the mass departure of inhabitants of the vicinity of the City of Mobile, Alabama, consists of a system of a primary and two secondary models (see Figure 2). The primary model is based on the entry of vehicles from the 20 on-ramps to I-65. The two secondary models assist the primary model with related traffic events such as car breakdowns and accidents, traffic control measures, interarrival signaling, and unforeseen emergency incidents, among others.

In the primary model, entering vehicles are created through a controlled wait-and-signal mechanism (see Figure 3). Attributes such as time of arrival, final destination exit, and accident incident proneness are established at this level. These attributes are assigned based on cumulative probability distributions generated by empirical data collected during the data acquisition phase of the project. When a car enters the highway, it is delayed by factors such as the travel distance and number of vehicles already on the road. The moving car keeps going through a loop of 


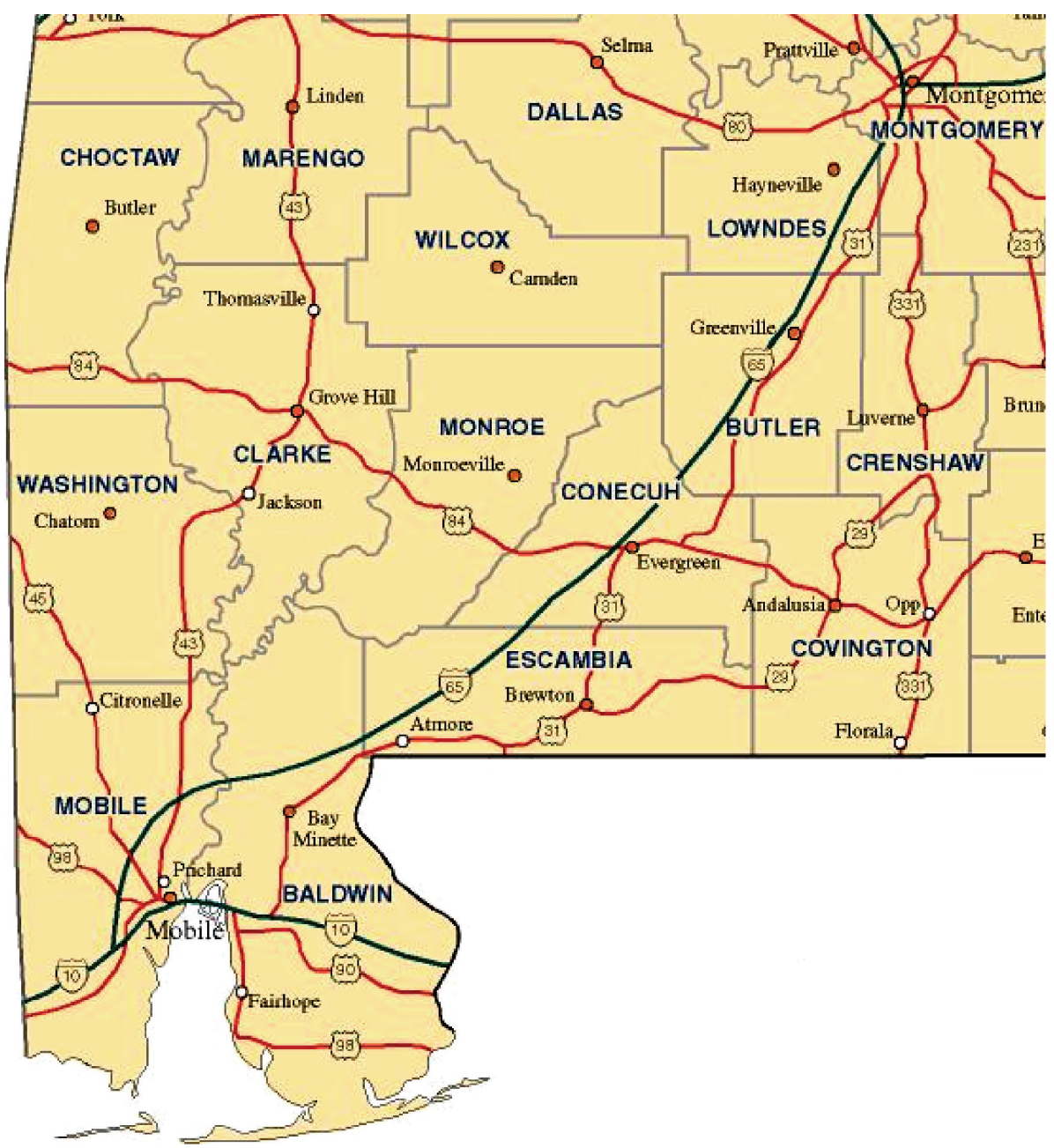

FIgURE 1: Counties locations.

High-level model flow

Primary flow
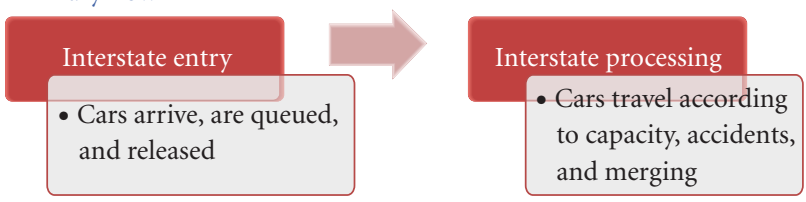

Secondary flow
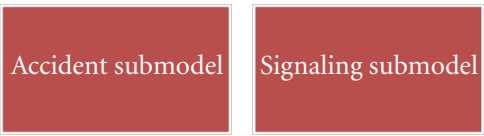

Figure 2: Primary and secondary simulated flows.

congested entries until its assigned exit attribute equals its final destination attribute. Once this loop sequence ends, the overall vehicle throughput and average travelling speed are then computed by the simulation. The primary model is equipped with a resolution factor variable. To prevent the system from growing beyond the software capabilities, the resolution factor was set to 25; thus, every moving entity within the system represents a pack of 25 vehicles, travelling bumper-to-bumper. Additionally, a maximum batch number of entering vehicles at each entry ramp was set based on the total amount people residing in the communities close to the corresponding ramp and the preestablished evacuation ratio. This evacuation ratio is an estimate of the fraction of the population exiting from a particular area as determined by ADOT officials. Other variables such as road length and number of lanes, average headway (i.e., the average distance between entities on the road), as well as lane occupancies are also used in the primary model to determine the time a vehicle spends on the highway during the evacuation process, the travelled distances, evacuation rates, average delays, and travelling speeds. For instance, the overall road occupancy level is increased according to the resolution factor and the rate of arrivals, and this leads to the computation of the overall delay experienced by drivers already travelling on the Interstate.

The secondary models that contribute to the primary model are the accident and signal models. The accident 


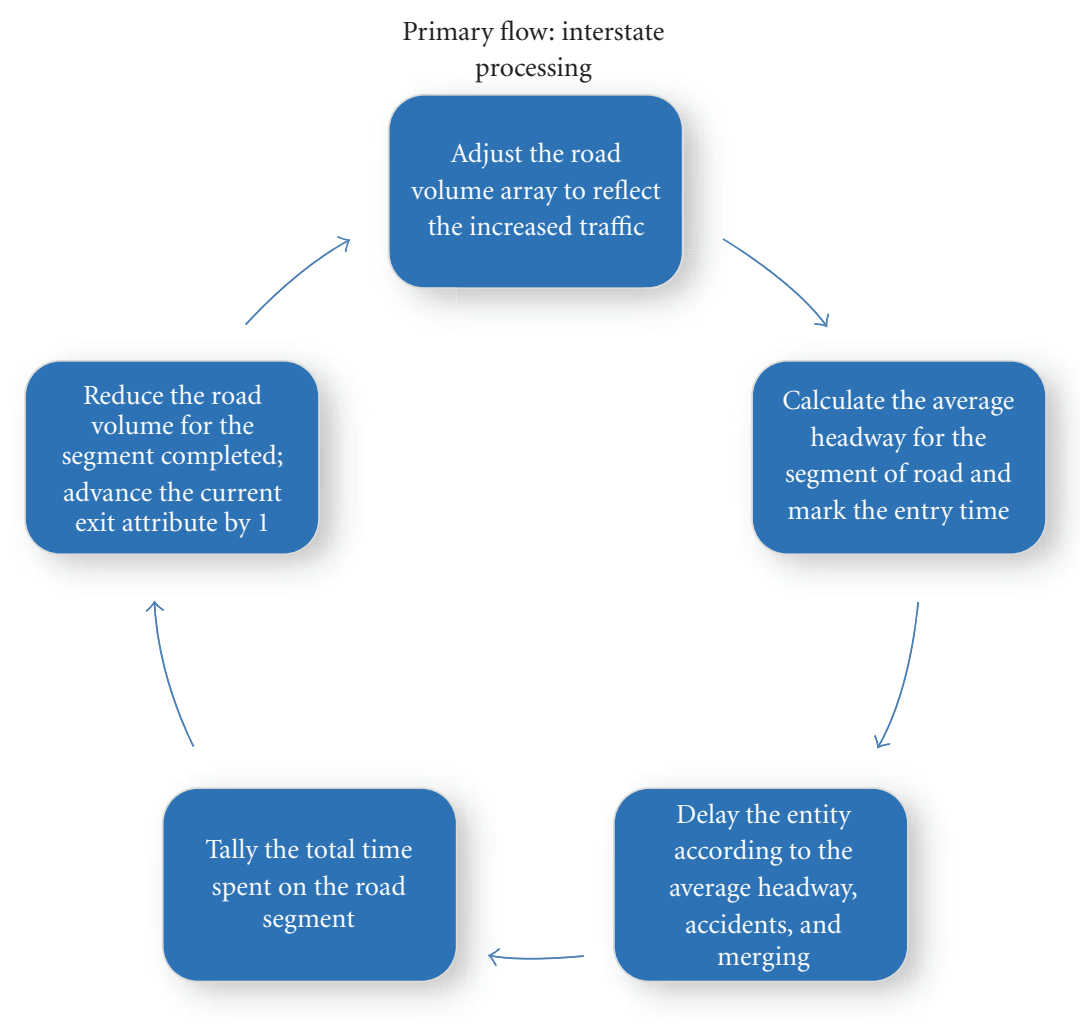

FIgURe 3: Primary model processing.

model dictates the frequency of accidents in the highway during the evacuation process. After the accident event is scheduled, a predetermined system delay becomes into effect in the model. The variables used in this secondary model, that is, the accident factor and the accident delay time, are generated through user-defined probability distributions emerging from the expertise of transportation officials. The other secondary model, the signal model, is in charge of timely signal of entities releases into the primary model from the entry ramps. This is a stochastic process defined by preestablished probability distributions (i.e., Poisson and Binomial distributions) as well as heuristics established by the project analysts. The key elements of the simulation model are discussed as follows.

4.1. Entity Creation. Figure 4 depicts the simulation logic for creating vehicles in the model. The traffic flow begins with the 20 ARRIVE blocks. Within each of these 20 ARRIVE blocks, the batch size is set equal to the number of entities that should be entering the interstate at each allowed time. This is done by dividing the number of cars that will be let on at each exit by the resolution factor, and then rounding the result to get the number of entities. For example, the fourth ARRIVE block has the following formula for batch size:

$$
\operatorname{ANINT}\left[\frac{\text { EntrRate(4) }}{\text { resfact }}\right] \text {. }
$$

"ANINT" is an internal system function which simply performs rounding. Next in the ARRIVE blocks, the rate at which these batches are created is set equal to the frequency at which the cars are released. This is accomplished by referencing the proper row in the SigDelay() array, which holds the three possible signaling frequencies to allow cars onto the interstate. Another array, ExitSig(), stores the signal (1, 2, or 3 ) that each car will respond to at each exit. For instance, the fourth ARRIVE block would have SigDelay(ExitSig(4)) as the formula for time between creations. This would reference the fourth row of the ExitSig () array and discover that cars at the fourth exit are signaled by Signal 2. The formula would then reference the second row in the $\operatorname{SigDelay}()$ array to find the proper time between creations.

The maximum number of batches that each of these 20 ARRIVE blocks creates is equal to the product of the total number of possible vehicles at the exit and the corresponding evacuation factor, divided by the resolution factor. This number is also rounded to be an interger. Hence, the formula corresponding to the 12th ARRIVE block, that is, 12th exit, is

$$
\operatorname{ANINT}\left[\frac{\text { TotalCars }(12) \times \operatorname{EvcRatio}(12)}{\text { resfact }}\right] \text {. }
$$

Within these ARRIVE blocks, the current time is marked in the attribute TimeIn, and the entry exit index is marked as ArrExit The attribute CurrExit is set equal to ArrExit, and is later used to advance entities along the interstate. The final attribute set at this point is OffExit, which gets its value from a discrete distribution with the help of the OffCumPr( () array. Before the simulation begins, the user 
Arena blocks: interstate entry

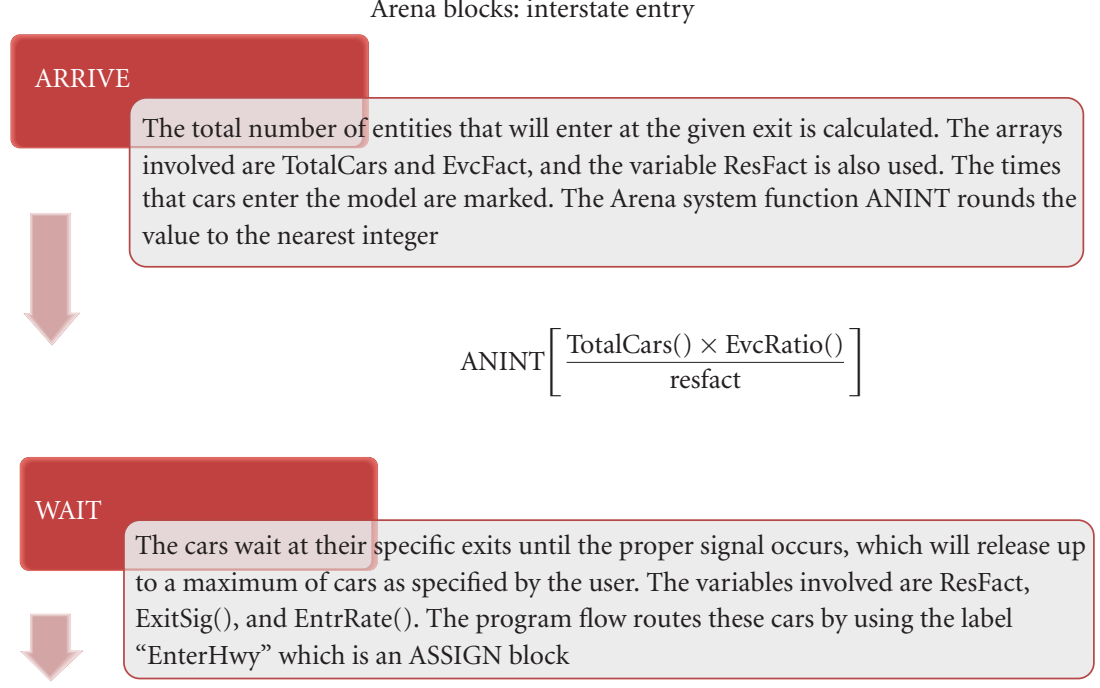

FIGURE 4: Interstate entry module.

accesses the Off-Exit Cumulative Probability array and inputs the cumulative probabilities that a car will exit at a given exit. OffExit references this array when drawing from the discrete distribution to find the entity's corresponding interstate exit. If, by chance, OffExit happens to be less than or equal to ArrExit, OffExit will be set to 20, that is, the last exit of the Interstate.

Entities then proceed to the WAIT block, where they are held until the proper signal for their exit occurs. The proper signal for the exit is found by referencing the ExitSig() array. For example, the 14th ARRIVE block, and thus the 14th WAIT block, would find its signal through the expression ExitSig(14). The release limit is set equal to the number of cars that will be released at each signal divided by the resolution factor.

4.2. Entity Processing. When an entity (i.e., vehicle) enters the interstate (refer to Figure 5), it goes to the first ASSIGN block on the upper left. First, the road volume array $\operatorname{RoadVol}()$, which stores the total number of cars, is adjusted upward by the resolution factor. Since the entity's current exit is stored in the attribute CurrExit, the road volume can be adjusted upward simply by the formula RoadVol(CurrExit) $=$ RoadVol(CurrExit) + resfact. After the road volume array compensates for the additional cars, the attribute AvHeadwy, which represents average headway, is calculated. Basically, this calculation takes the length of the road, multiplies it by the number of lanes on the road, and subtracts out the length of road that all the cars are using (assuming an average car length is $15 \mathrm{ft}$ ). All of this is then divided by the length of road that the cars are using to ultimately come out with the average headway:

$$
\frac{[\mathrm{RL}(\mathrm{CE}) \times \text { RoadLane }(\mathrm{CE}) \times 5280]-[\operatorname{RoadVol}(\mathrm{CE}) \times 15]}{\operatorname{RoadVol}(\mathrm{CE}) \times 15},
$$

where $\mathrm{RL}(\mathrm{CE})$ is the road length of the current exit.
The constant 5,280 represents a unit conversion from miles to feet, and the constant 15 represents the average car length. Finally, the time in to the current exit, ExTimeIn, is marked so that the total time spent on the current strip of road can be calculated.

Next, the time to be spent on the current road segment is calculated in the DELAY block. The formula references a table called SpeedTbl, which uses the assumption that a linear relationship exists between average headway and speed $(1 \mathrm{car}$ length $=10 \mathrm{mph})$, and that the maximum speed is $70 \mathrm{mph}$. Table functions in Arena will automatically interpolate; so when an average headway of $2.37 \mathrm{ft} / \mathrm{car}$ is used as an input, the function will output $23.7 \mathrm{mph}$. The total time a car spends on the current strip of road is calculated as follows:

$$
\frac{\mathrm{RL}(\mathrm{CE}) \times 60 \times \operatorname{AccFact}(\mathrm{CE}) \times \operatorname{MrgFact}(\mathrm{CE})}{\mathrm{TF}(\text { SpeedTbl, AvHeadwy })} .
$$

The constant of 60 is present for unit conversion purposes, in that 1 hour $=60$ minutes. AccFact () and $\operatorname{MrgFact}()$ are arrays that contain values between 0 and 1 which will slow the traffic proportionally according to the factors they present. For instance, if the 16th exit had an accident, AccFact(16) would be set to 0.6 to reflect that the accident is slowing traffic down to $60 \%$ of what it would be otherwise. Additionally, if the ninth exit is left open for cars to enter the Interstate, MrgFact(9) would likely be set equal to a number less than 1 , whereas closed exits would maintain a factor of 1 since merging would not be affecting traffic at closed exits. "TF" is a system function of Arena that stands for "Table Function." Unit analysis shows that the final result is minutes, as the top is miles $*$ minutes/hour and the bottom is miles/hour.

The next ASSIGN block adjusts the road volume downward as the entity has now completed the current strip of road. The TALLY block then records the total time spent on the specific exit. The next ASSIGN block advances CurrExit 
Arena blocks: interstate processing

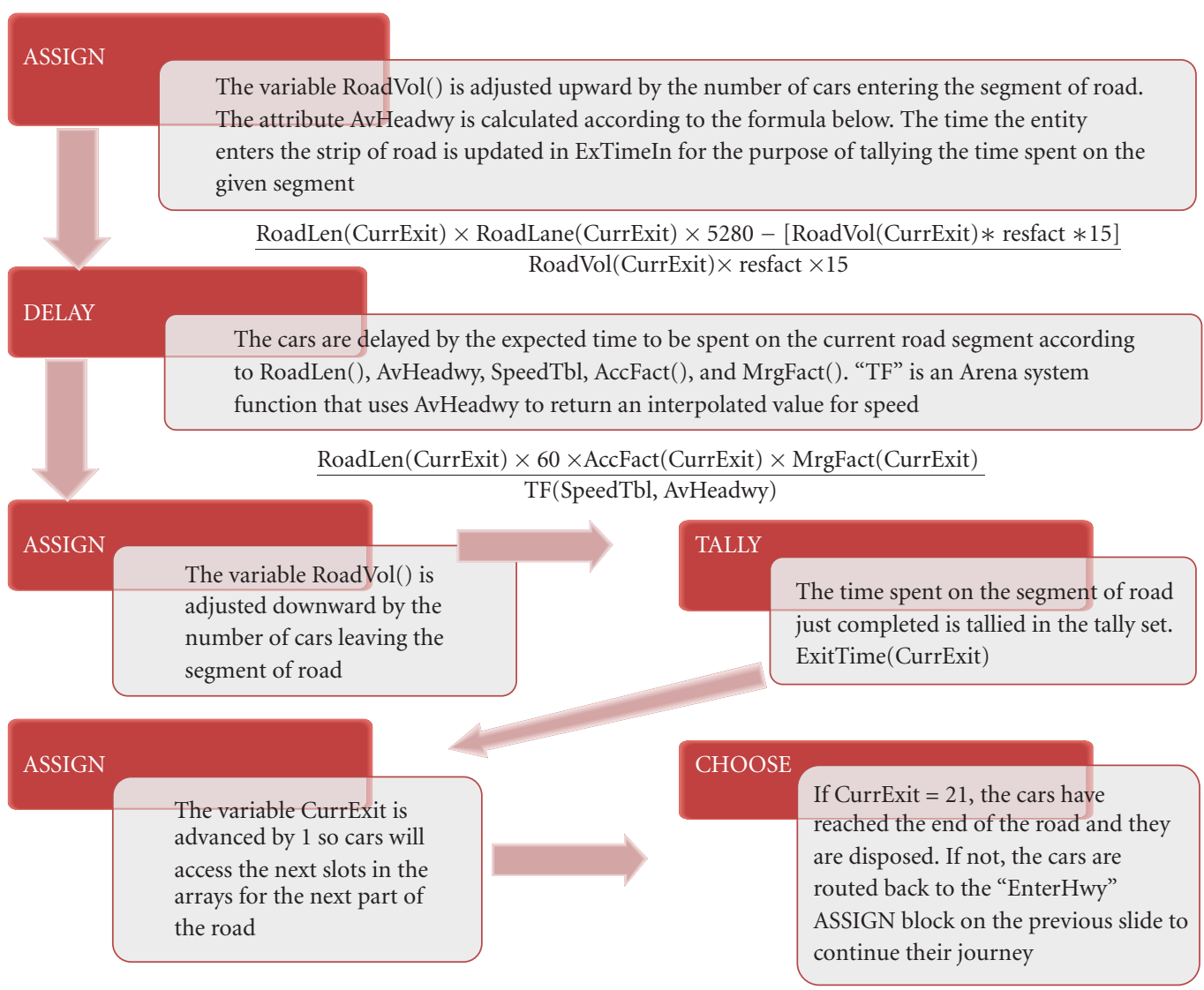

FIGURE 5: Interstate processing module.

by 1 as the entity has completed the strip of road and is now advancing to the next segment. Finally, the CHOOSE block checks to see whether the entity has reached the exit that will leave the interstate (if CurrExit = OffExit) or to see if the car has reached Montgomery (if CurrExit $=21$ ). If either of those conditions is true, the entity is disposed. Otherwise, the entity is routed back around to the first ASSIGN block in the upper left corner where the next segment of road has its road volume incremented upward.

4.3. Traffic Accidents Modeling. Dummy entities, which represent accidents, are created at a set time interval (in minutes) stored in a single variable called AccFreq. For each simulated accident event, an ASSIGN block allocates the exit where the accident is to occur (AccExit) according to a userdefined probability distribution named $\operatorname{Acc} C u m \operatorname{Pr}()$ as well as a factor to represent the resulting slower traffic (AccFact). Under ideal conditions, AccFact is equal to 1 for any give road segment; however, after the ASSIGN block determines that an accident has happened at a particular exit, the respective AccFact is reset to a number smaller than 1 (e.g., 0.7) based on the probabilistic equations embedded in the model. The accident is allowed to persist for a certain duration of time, AccRcvr. After that time has elapsed, the accident factor for that specific exit is returned to one. Figure 6 depicts the logic embedded in the simulation's Accident Submodel.

\section{Simulation Runs}

5.1. Initial Model. Figure 4 depicts a snapshot of the actual Arena simulation model created in this study. With parameters set for the initial model, that is, all 20 ramps open, the simulation was run for a twelve-hour period. The resolution factor, that is, the entity to car ratio, was set to 25 , and the entry rates for each on-ramp were set according to the total amount of vehicles as determined with the data presented in Tables 1 and 2. For this initial model, a greater number of vehicles were allowed to get on the Interstate closer to the coast than at other points located further upstream. Hence, such on-ramps experienced a faster rate of signals for the releasing of batches of vehicles. The final destination attribute for each simulated vehicle was established through a probably scheme based on the assumption that there is an equal chance (i.e., 20\%) for a travelling car to exit the interstate at any of the five exit ramps of I-65 in the City of Montgomery. Test statistics were collected from simulation after 30 independent replications. The average number of vehicles that reached their final destination during any 12hour period was 113,318 . That is, with all on-ramps open for 
Arena blocks: accident submodel

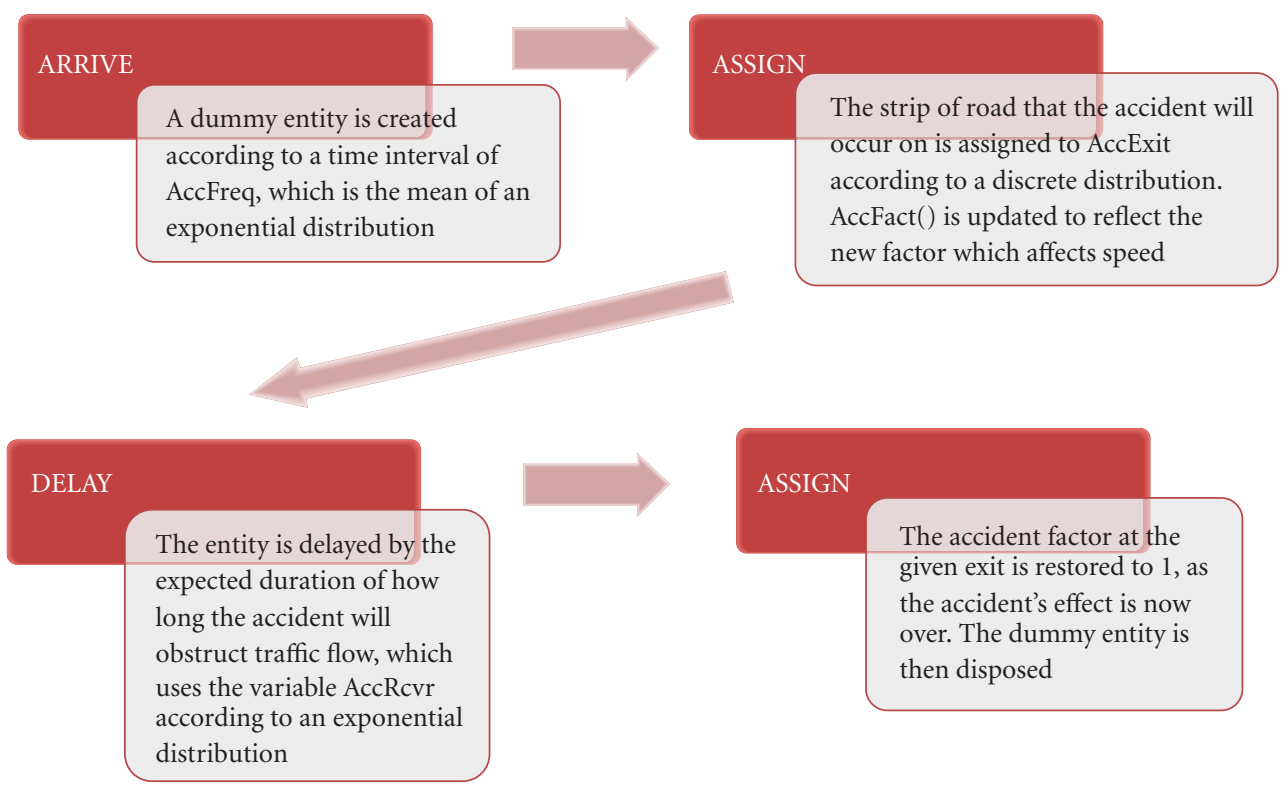

Figure 6: Accident submodel.

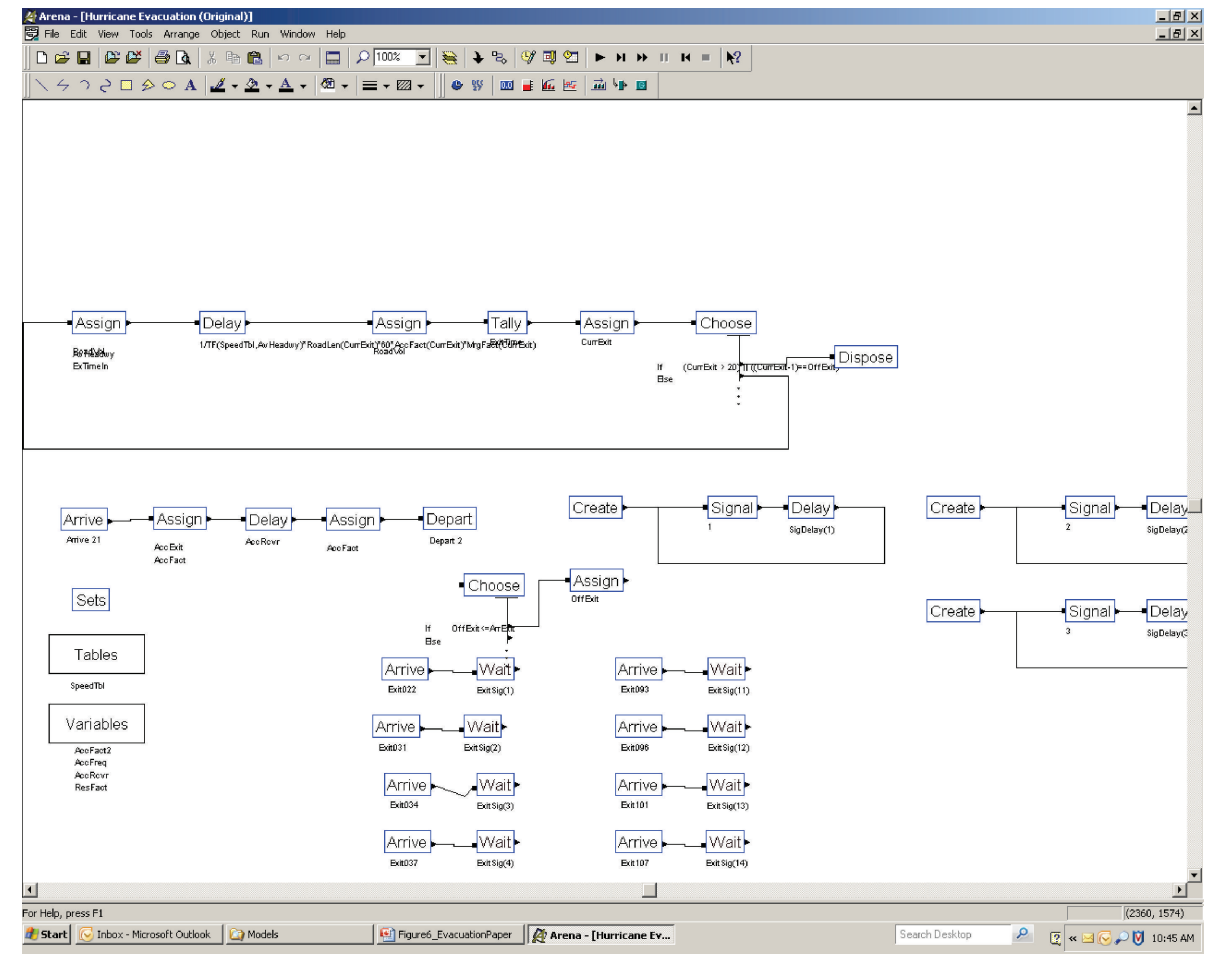

FIgURE 7: Hurricane evacuation model.

traffic evacuation, the average evacuation rate was 9,442.17 vehicles/hour (Figure 7).

5.2. Evaluation of Alternative Models. The main focus on the study was to investigate that effect that closing on-ramps from entering traffic had on the overall evacuation process of the Alabama Gulf region during a hurricane situation.
The closing of on-ramps was modeled by redirecting coming vehicles from the arrival at the closed on-ramp to subsequent entry points further north, taking into consideration the time of travel through side road, the availability of these side roads, as well as the open on ramps' own traffic volumes. This traffic rerouting was carried out through a random variable distribution of vehicles to on-ramps located no further than 
TABLE 3: Two sample T means comparisons.

\begin{tabular}{|c|c|c|c|c|c|c|}
\hline Identifier & $\begin{array}{c}\text { ESTD. mean } \\
\text { difference }\end{array}$ & $\begin{array}{l}\text { Standard } \\
\text { deviation }\end{array}$ & $\begin{array}{l}0.950 \text { C.I. } \\
\text { half-width }\end{array}$ & Minimum value & Maximum value & Number obs \\
\hline \multicolumn{7}{|c|}{ With Exits 130 and 57 Closed: } \\
\hline \multirow{2}{*}{ Cars } & \multirow{2}{*}{135} & \multirow{2}{*}{136} & \multirow{2}{*}{273} & $4.01 e+003$ & $6.88 \mathrm{e}+003$ & 30 \\
\hline & & & & $3.87 \mathrm{e}+003$ & $5.55 e+003$ & 30 \\
\hline \multicolumn{7}{|c|}{ With Exit 142 Closed: } \\
\hline \multirow{2}{*}{ Cars } & \multirow{2}{*}{-13.6} & \multirow{2}{*}{131} & \multirow{2}{*}{262} & $4.01 \mathrm{e}+003$ & $6.88 \mathrm{e}+003$ & 30 \\
\hline & & & & $3.97 e+003$ & $5.57 e+003$ & 30 \\
\hline \multicolumn{7}{|c|}{ With Exit 128 Closed: } \\
\hline \multirow{2}{*}{ Cars } & \multirow{2}{*}{-39.7} & \multirow{2}{*}{145} & \multirow{2}{*}{290} & $4.01 \mathrm{e}+003$ & $6.88 \mathrm{e}+003$ & 30 \\
\hline & & & & $3.92 \mathrm{e}+003$ & $6.41 \mathrm{e}+003$ & 30 \\
\hline \multicolumn{7}{|c|}{ With Exit 54 Closed: } \\
\hline \multirow{2}{*}{ Cars } & \multirow{2}{*}{56.1} & \multirow{2}{*}{138} & \multirow{2}{*}{276} & $4.01 e+003$ & $6.88 \mathrm{e}+003$ & 30 \\
\hline & & & & $3.79 e+003$ & $5.46 e+003$ & 30 \\
\hline
\end{tabular}

Fail to reject $\mathrm{H} 0 \Rightarrow$ Means are equal at 0.05 level

thirty miles away from the closed entry point. This was a combinatorial procedure consisting of two phases. The first step was the analysis of twenty simulation models each pertaining to the closing of a particular on-ramp in the model. The next step was to simulate the closing of multiple on-ramps through permutations of two and three on-ramps closing at a time.

To guarantee statistical soundness of the Hypothesis Testing procedure, all simulation trials involved 12 hours of simulated time, each replicated 30 times. The Null Hypothesis of the study implied that the means for the evacuation rate remained the same for the original model with all 20 entry ramps open, and for every other permuted model under examination. The hypothesis testing was performed in a pairwise fashion, that is, original model versus alternate model, and an alpha level of 5\% was used in all tests.

Table 3 displays the most relevant results from the statistical analysis described before. The authors found that there are five on-ramps that can be strategically controlled by safety and traffic officials during an evacuation situation without slowing down the overall mass departure of residents. These on-ramps are Exit 54, Exit 57, Exit 128, Exit 130, and Exit 142. Exits 54, 128, and 142 can be shut down from entering traffic individually without affecting of overall evacuation rate of vehicles. When closed individually, exits 57 and 130 posed a detrimental effect on the overall evacuation effort; however, it is interesting to note that when they are closed at the same time, their combined effect does not alter the overall flow of evacuating traffic on the Interstate.

\section{Conclusion}

Alabama traffic officials and emergency personnel want to have the most effective way of ensuring the safety of coastal residents when the danger of severe tropical weather is eminent. Through the study, a comprehensive simulation of I-65 was conducted to access its effectiveness as potential evacuation route during a hurricane situation.
It was discovered that by having all 20 on-ramp exits open to merging vehicles bound north, an average of 113,318 vehicles will reach the City of Montgomery safely within a 12-hour period. However, having all exits blocked from traffic bound south poses great difficulty to emergency-response officials needing to access areas in the path of the storm. Through a rigorous and exhaustive process it was determined that five of the twenty ramps can be strategically controlled to resolve conflicts when restricted flow of vehicles bound south on the Interstate shoulder is needed. The findings of this study serve as evidence for the need of similar studies to be conducted in other main routes of evacuation along the coastal areas of the United State.

Finally, the authors have provided a simulation platform that has proven effective to analyze discrete networks presenting a flow pattern in equilibrium. The model described in this paper can thus be generalized to similar systems (e.g., communication and/or distribution networks) where the impact of having multiple available entry ports is not fully comprehended.

\section{References}

[1] B. Gerdes, "16 Percent of Alabamians Live in Hurricane Counties, According to UA's State Data Center, NOAA," The University of Alabama News, 2007, http:// uanews.ua.edu/anews2007/jun07/counties061307.htm.

[2] A. Pal, M. Triche, A. Graettinger, K. Rao, J. McFadden, and D. Turner, "Enhancements to emergency evacuation procedures," Final Research Report, University of Alabama Transportation Center, 2005.

[3] ALDOT Maintenance Bureau, "I-65 Reverse-Laning for Hurricane Evacuation," 2007, http://www.dot.state.al.us/Docs/ Bureaus/Maintenance/I-65+REVERSE-LANING+FOR +HURRICANE+EVACUATION.htm.

[4] V. Sisiopiku, "Development of Dynamic Traffic Assignment Model to Evaluate Lane Reversal Plans for I-65," UAB School of Engineering, 2007, http://main.uab.edu/ soeng/Templates/Inner.aspx?pid=98705. 
[5] L. J. LeBlanc, "An algorithm for the discrete network design problem," Transportation Science, vol. 9, no. 3, pp. 183-199, 1975.

[6] H. Yang and M. G. H. Bell, "Models and algorithms for road network design: a review and some new developments," Transport Reviews, vol. 18, no. 3, pp. 257-278, 1998.

[7] R. S. Solanki, J. K. Gorti, and F. Southworth, "Using decomposition in large-scale highway network design with a quasioptimization heuristic," Transportation Research Part B, vol. 33, no. 2, pp. 127-140, 1998.

[8] T. L. Magnanti and R. T. Wong, "Network design and transportation planning: models and algorithms," Transportation Science, vol. 18, no. 1, pp. 1-55, 1984.

[9] J. G. Wardrop, "Some theoretical aspects of road traffic flow research," Proceeding of the Institute of Civil Engineer II, vol. 1, pp. 325-378, 1952.

[10] R. Steinberg and W. I. Zangwill, "The prevalence of Braess' paradox," Transportation Science, vol. 17, no. 3, pp. 301-318, 1983.

[11] S. Tung, Designing optimal networks: a knowledge-based computer aided multicriteria approach, Ph.D. dissertation, University of Washington, Seattle, Wash, USA, 1986.

[12] T. L. Friesz and S. Shah, "An overview of nontraditional formulations of static and dynamic equilibrium network design," Transportation Research Part B, vol. 35, no. 1, pp. 521, 2001.

[13] E. Turban and J. Aronson, Decision Support Systems and Intelligent Systems, Prentice-Hall, Upper Saddle River, NJ, USA, 2001.

[14] F. Hillier and G. Lieberman, Introduction to Operations Research, McGraw-Hill, New York, NY, USA, 8th edition, 2005.

[15] J. D. Murchland, "Braess's paradox of traffic flow," Transportation Research, vol. 4, no. 4, pp. 391-394, 1970.

[16] J. Cohen and F. Kelly, "A paradox of congestion in a queuing network," Journal of Applied Probability, vol. 27, pp. 730-734, 1990.

[17] T. Bass, "Road to ruin," Discover, pp. 56-61, 1992.

[18] D. J. Fonseca, S. Daosuparoch, G. P. Moynihan, and D.-S. Chen, "A computer-based system for road selection," Expert Systems, vol. 20, no. 3, pp. 133-140, 2003.

[19] K. C. Laudon and J. P. Laudon, Management Information Systems, McGraw-Hill, Upper Saddle River, NJ, USA, 5th edition, 2007.

[20] Alabama Department of Transportation, "Traffic Counts Database," 2007, http://aldotgis.dot.state.al.us/atd/default .aspx.

[21] W. D. Kelton, R. P. Sadowski, and D. T. Sturrock, Simulation with Arena, Prentice Hall, New York, NY, USA, 4th edition, 2007. 

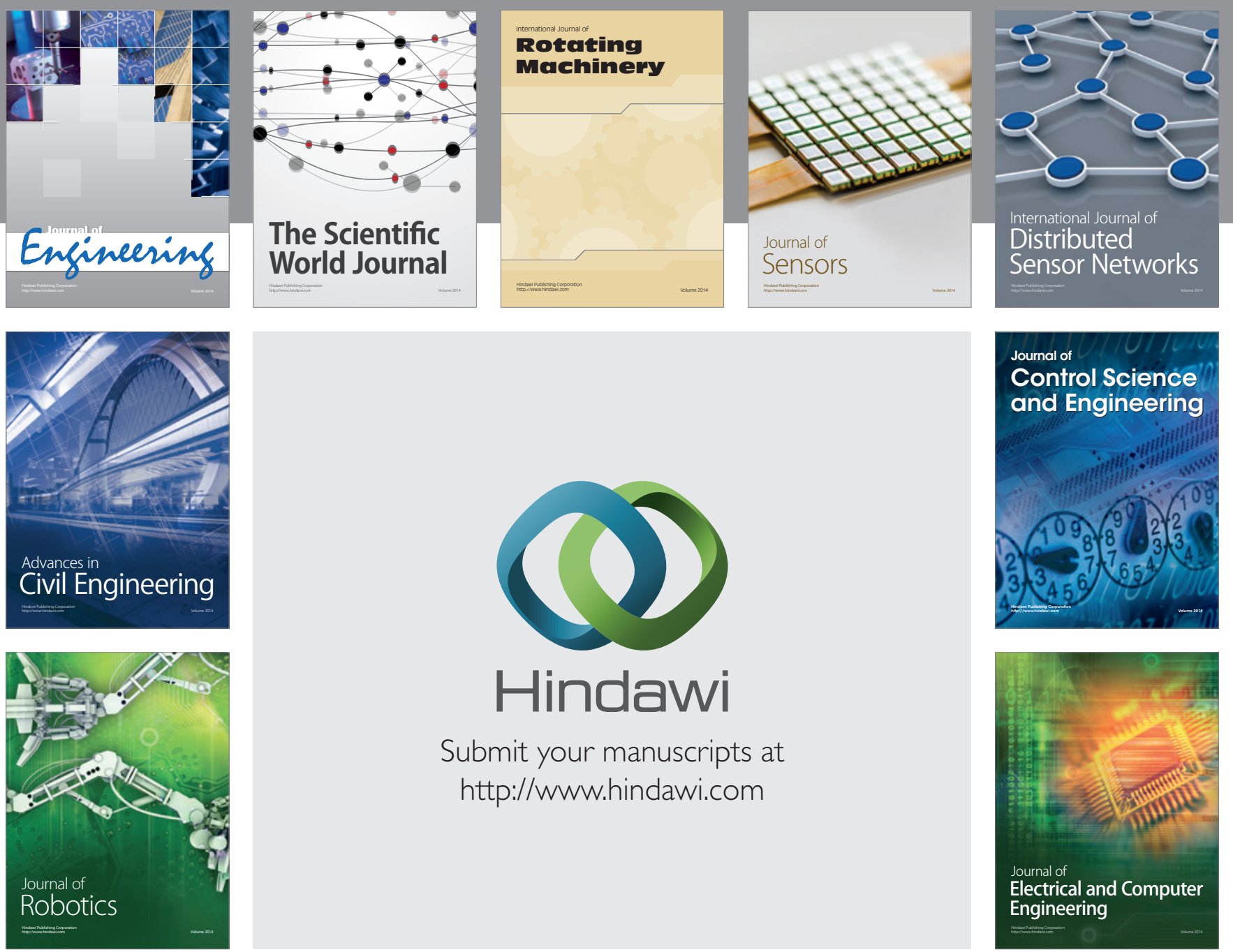

Submit your manuscripts at

http://www.hindawi.com
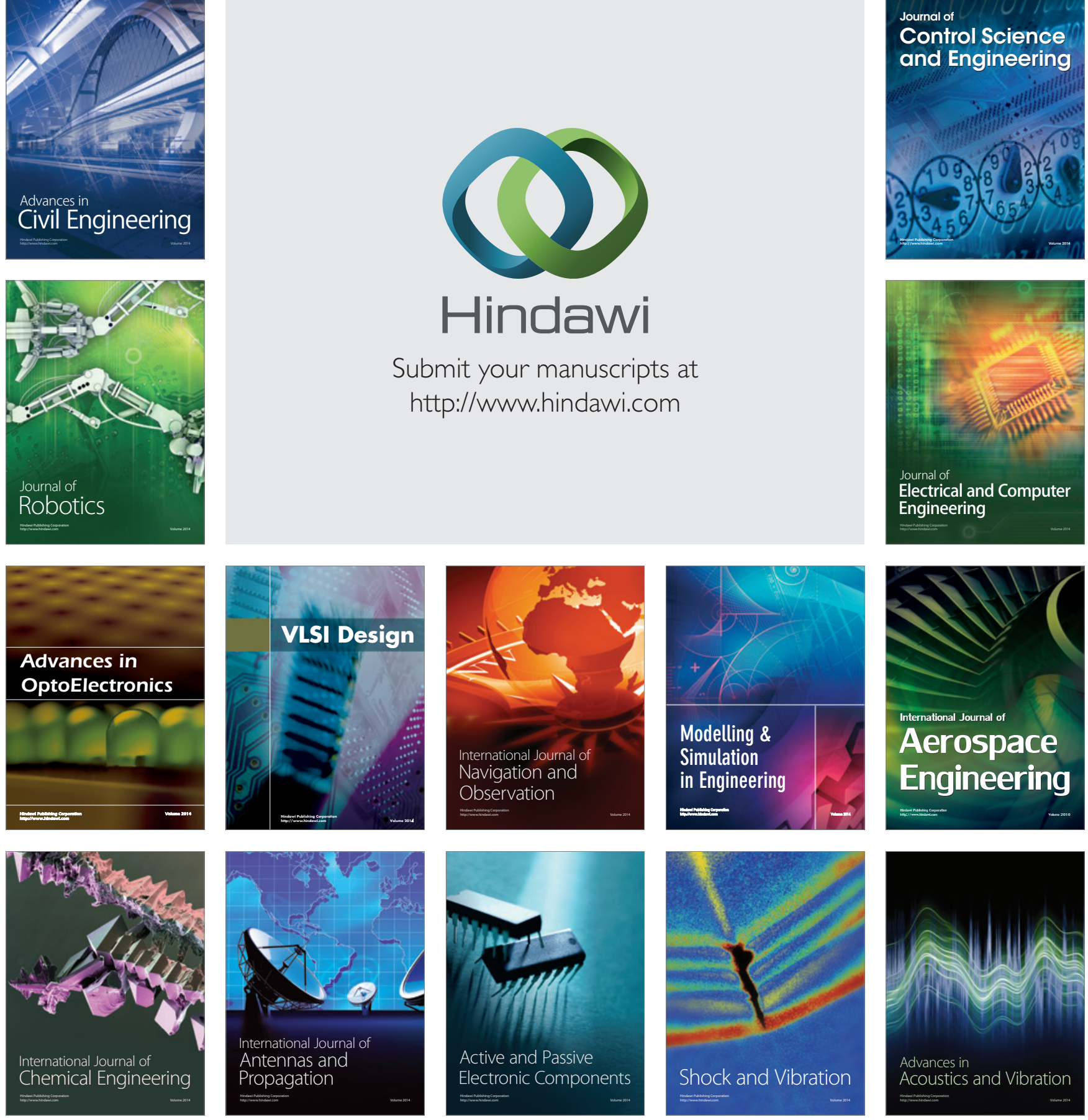